IMPACT MITIGATION USING KINEMATIC CONSTRAINTS AND THE FULL SPACE

DARAMETERIZATION METHOD*

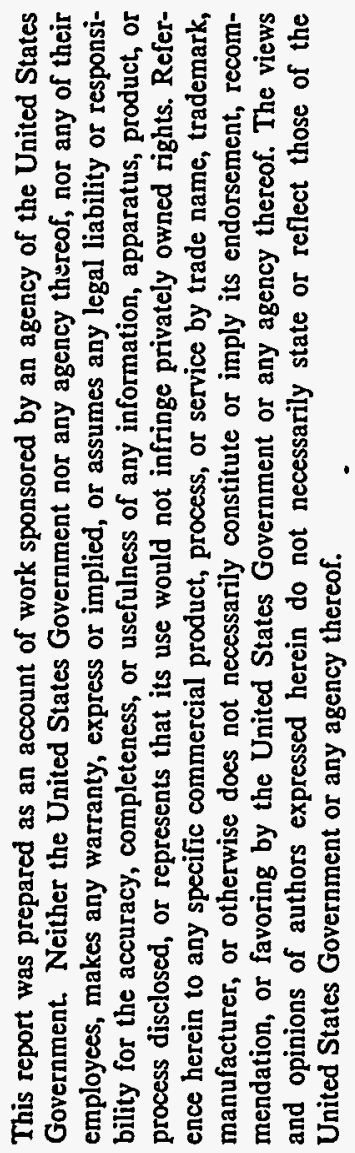

\author{
Kristi A. Morgansen and François G. Pin \\ Robotics and Process Systems Division \\ Oak Ridge National Laboratory \\ P.O. Box 2008 \\ Oak Ridge, TN 37831-6305
}

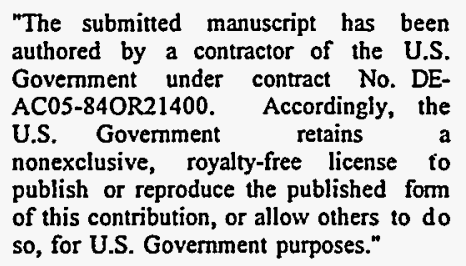

authored by a contractor of the US.

Government under contract No. DE-

AC05-84OR21400. Accordingly, the

U.S. Government retains a

publish or reproduce the published form

so, for U.S. Government purposes."

Submitted to: 1996 IEEE International Conference on Robotics and Automation, Minneapolis, Minnesota, April 22-28, 1996

*This research was supported in part by the U.S. Air Force Combat Command (ACC), the U.S. Air Force Munition Material Handling Equipment (MMHE) Focal Point, the U.S. Air Force Reliability and Maintainability Technology Insertion Program (PRAM-RAMTIP), the U.S. Department of Defense, Office of the Secretary of Defense (OSD), and the U.S. Advanced Project Research Agency (ARPA), under Interagency Agreement 2146-H055-A1 between the U.S. Air Force Material Command (AFMC) San Antonio Air Logistics Center, Robotics and Automation Center of Excellence (SA/ALC-RACE) and the U.S. Department of Energy under contract DE-ACO5-84OR21400 with Lockheed Martin Energy Systems, Inc., in part by the DOE-sponsored Professional Internship Program administered by Oak Ridge Associated Universities, and in part by a National Science Foundation Graduate Research Fellowship. 


\title{
Impact Mitigation Using Kinematic Constraints and the Full Space Parameterization Method ${ }^{\dagger}$
}

\author{
Kristi A. Morgansen and François G. Pin \\ Oak Ridge National Laboratory \\ PO Box 2008 \\ Oak Ridge, TN 37831-6305
}

\begin{abstract}
A new method for mitigating unexpected impact of a redundant manipulator with an object in its environment is presented. Kinematic constraints are utilized with the recently developed method known as Full Space Parameterization (FSP). System performance criterion and constraints are changed at impact to return the end effector to the point of impact and halt the arm. Since large joint accelerations could occur as the manipulator is halted, joint acceleration bounds are imposed to simulate physical actuator limitations. Simulation results are presented for the case of a simple redundant planar manipulator.
\end{abstract}

\section{Introduction}

In many standard robot tasks, the robot must be able to touch objects in its environment without damaging itself or the objects. Examples of such tasks are peg-in-hole insertions [10], biped walking [13], and surface following [12]. The work in this paper concerns the problem of trajectory planning for redundant manipulators whose end effectors have contacted an unexpected surface.

Much of the work that has been done with respect to impact has approached the problem with the goal

\footnotetext{
tThis research was supported in part by the U.S. Air Force Combat Command (ACC), the U.S. Air Force Munition Material Handling Equipment (MMHE) Focal Point, the U.S. Air Force Reliability and Maintainability Technology Insertion Program (PRAM-RAMTIP), the U.S. Department of Defense, Office of the Secretary of Defense (OSD), and the U.S. Advanced Project Research Agency (ARPA), under Interagency Agreement 2146-H055-A1 between the U.S. Air Force Material Command (AFMC) San Antonio Air Logistics Center, Robotics and Automation Center of Excellence (SA/ALC-RACE) and the U.S. Department of Energy under contract DE-AC05840R21400 with Lockheed Martin Energy Systems, Inc.; in part by the DOE-sponsored Professional Internship Program administered by Oak Ridge Associated Universities, and in part by a National Science Foundation Graduate Research Fellowship.
}

of minimizing the force of impact (see [2],[9]) or relating the force of impact to the manipulator configuration at impact (see [11]). Work on modeling impact has been presented in [9], [11], [12] and [14]. Some of the research that has been done to utilize the benefits of redundancy to improve impact conditions can be found in [3], [7], and [11].

Our work is fundamentally different from the approaches which are built around the force of impact. These methods for the most part are based on controlling the dynamics of the system, whether or not it has redundancy. In contrast, we deal with impact from a completely kinematics-oriented viewpoint. Also, our method does not assume that the time or location of impact is known before it occurs as assumed in [7], [11] and [13]. Although our approach to impact mitigation can easily be implemented for nonredundant systems, we are more interested in applications to redundant manipulators (for a review of some approaches to redundancy resolution see [5], [8]). The goal is to utilize the manipulator redundancy to mitigate the post-impact behavior of the system since we consider that the impact is unexpected. The method which we use to perform the inverse kinematics calculations is the recently developed Full Space Parameterization (FSP). A thorough treatment of this method can be found in [1], [4], and [6]. As has been done in much of the previous work on impact, we will treat impact as a transition between one type of motion and another. In our case, the transition will cause a switching from one set of constraints to a second set to be used in the optimization of a cost function. Since we are using kinematics rather than dynamics, we will be looking at impact mitigation on the level of joint behavior rather than focusing on directly controlling the force of impact at the end effector.

One of the dominating goals of our approach is to minimize the distance that the end effector moves into the surface which it has contacted. As discussed in [3], 
this approach is similar to minimizing the post-impact forces since the less the end effector penetrates the object, the smaller the resulting forces will be.

Before impact, the manipulator is free to move about the workspace using whatever motion criterion and task constraints are desired. When impact occurs, we would like the manipulator to discontinue its trajectory and halt the end effector at the point of impact. In order to produce realistic behavior after impact, each joint must obey acceleration limits. This will prevent the joints from using unacceptably high accelerations to return the end effector to the point of impact and halt all joint motion. The presence of redundancy will allow for minimal displacement of the end effector (using nullspace motions) while the joints slow to a stop.

The remainder of the paper is organized as follows. Section two will present a basic discussion of the FSP method. Section three gives the criterion and constraints that will be used on the manipulator after impact has occurred. Also, the complete algorithm that is used to halt the manipulator after impact is given and discussed. Section four presents the results of simulated impact for a simple redundant planar manipulator.

\section{Overview of Full Space Parameteri- zation}

For any robotic manipulator system, the forward kinematics are usually described by the equation

$$
\bar{x}=F(\bar{q})
$$

where $\vec{x}$ is the location of the manipulator in the world coordinate system, $\bar{q}$ is the vector of joint angles measured in local coordinate frames, and $F(\cdot)$ is the transformation function. In general, desired motions are expressed as trajectories in end effector space. These trajectories are broken up into finite steps of length $\Delta \bar{x}$. The relationship between end effector steps $\Delta \bar{x}$ and joint space steps $\Delta \bar{q}$ is found by differentiating and linearizing Eq. 1

$$
\frac{\Delta \bar{x}}{\Delta t}=\mathrm{J}_{\Delta t} \frac{\Delta \bar{q}}{\Delta t}
$$

where $\mathbf{J}_{\Delta t}$ is the linearized system Jacobian. In the work that follows, a uniform time step of $0.0333 \mathrm{~s}$ will always be assumed. The equation with which we will be working is then

$$
\Delta \bar{x}=\mathfrak{J} \Delta \bar{q} .
$$

In order to carry out trajectories, the robot must be given motions in terms of joint space variables. This task requires some type of inverse transformation to be made to convert from the known quantity $\Delta \bar{x}$ to the desired quantity $\Delta \bar{q}$.

The FSP method has been specifically designed to optimally solve the inverse kinematics problem for redundant systems in the presence of applied constraints and behavioral criterion. For a redundant system, $\mathbf{J}$ will have fewer rows $n$ than columns $m$, and the number of vectors $\Delta \bar{q}$ which satisfy Eq. 3 will typically be infinite in number. This infinite set of solution vectors forms a subspace of a larger space which is spanned by $m-n+1$ linearly independent solution vectors $\bar{g}_{k}$ each of which satisfies the equation

$$
\Delta \bar{x}=\mathrm{J} \bar{g}_{k} .
$$

The vectors $\bar{g}_{k}$ can easily be found by inverting square submatrices $\mathbf{J}_{k}$ of the Jacobian $\mathbf{J}$ and inserting a 0 into the components of $\bar{g}_{k}$ corresponding to the columns of $\mathbf{J}$ that were removed to form $\mathbf{J}_{k}$. The proof for existence of and algorithms for the determination of the $m-n+1$ linearly independent solution vectors $\bar{g}_{k}$ can be found in [1], [4], and [6].

Once the $m-n+1$ solution vectors $\bar{g}_{k}$ have been found, the solution space, $\mathcal{S}$, of Eq. 4 is given by (see [6])

$$
\mathcal{S}=\left\{\Delta \bar{q} \mid \Delta \bar{q}=\sum_{i=1}^{m-n+1} t_{i} \bar{g}_{i}, \sum_{i=1}^{m-n+1} t_{i}=1\right\} .
$$

If the manipulator motion is to be in the nullspace, then the condition $\sum t_{i}=1$ must be replaced by the condition $\sum t_{i}=0$. The specific solutions optimizing a criterion $Q\left(\Delta \bar{q}\left(t_{i}\right)\right)$ and satisfying a set of $r$ constraints $C^{j}=0$ are found by minimizing the Lagrangian

$L\left(t_{i}, \mu, \nu_{j}\right)=Q\left(t_{i}\right)+\mu\left(\sum_{i=1}^{m-n+1} t_{i}-1\right)+\sum_{j=1}^{r} \nu_{j} C^{j}\left(t_{i}\right)$

where $\mu$ and $\nu_{j}$ are Lagrange multipliers. If the general criterion can be expressed as

$$
Q=\left\|\Delta \bar{Z}(\bar{q}, \Delta \bar{q})-\ddot{Z}_{r}\right\|^{2}, \quad \Delta \bar{Z}=B(\bar{q}) \Delta \bar{q}
$$

where $B(\bar{q})$ is a matrix, and the constraints $C^{j}$ can be written as

$$
\bar{\beta}^{j^{T}} \bar{t}-1=0, \quad j=[1, r]
$$

then the optimality conditions become

$$
\left\{\begin{array}{c}
G \bar{t}+\bar{H}+\mu \bar{e}+\sum_{i=1}^{r} \nu_{i} \bar{\beta}^{i}=0 \\
\bar{e}^{T} \bar{t}=1 \\
\bar{\beta}^{j^{T}} \bar{t}=1, \quad j=[1, r]
\end{array}\right.
$$


with

$$
\begin{array}{ccc}
\bar{H}, & H_{k}=\Delta \bar{Z}_{r}^{T} B \bar{g}_{k}, & k=[1, m-n+1] \\
G, & G_{i j}=\bar{g}_{i}^{T} B^{T} B \bar{g}_{j}, & i, j=[1, m-n+1] \\
\bar{e}, & e_{i}=1, & i=[1, m-n+1]
\end{array}
$$

Solving these equations gives (see [6])

$$
\begin{aligned}
\bar{\nu} & =A^{-1}\left(a \bar{d}-\bar{b}\left(1+\bar{e}^{T} G^{-1} \bar{H}\right)\right) \\
\mu & =-\left(1+\bar{\nu}^{T} \bar{b}+\bar{e}^{T} G^{-1} \bar{H}\right) / a \\
\bar{t} & =-G^{-1}(\mu \bar{e}+\mathcal{B} \bar{\nu}+\bar{H})
\end{aligned}
$$

for non-nullspace motion, and for nullspace motion Eqs. 13 and 14 are replaced by

$$
\begin{aligned}
\vec{\nu} & =A^{-1}\left(a \bar{d}-\bar{b}^{T} \bar{e}^{-1} \bar{H}\right) \\
\mu & =-\left(\bar{e}^{T} G^{-1} \bar{H}+\bar{\nu}^{T} \bar{b}\right)
\end{aligned}
$$

where

$$
\begin{gathered}
a=\bar{e}^{T} G^{-1} \bar{e} \\
\bar{b}, \quad b_{i}=\bar{e}^{T} G^{-1} \bar{\beta}^{i}=\bar{\beta}^{i^{T}} G^{-1} \bar{e} \quad i=[1, r] \\
\bar{d}, \quad d_{i}=1+\bar{\beta}^{i^{T}} G^{-1} \bar{H} \quad i=[1, r] \\
A, \quad A_{i j}=b_{i} b_{j}-a \bar{\beta}^{i^{T}} G^{-1} \bar{\beta}^{j} \quad i=[1, r], j=[1, r]
\end{gathered}
$$

and $\mathcal{B}$ is a matrix whose columns are $\bar{\beta}^{i}$.

\section{Constraints and Algorithm}

In order to use FSP and a kinematics approach to impact mitigation, appropriate constraints and criterion must be developed to produce the desired system behavior after impact has occurred. In this paper we would like the trajectory after impact to evolve such that the end effector is slowed and returns to the point of impact while all of the joints stay within specified acceleration bounds and are slowed to a halt. In order to produce a realistic behavior, bounded acceleration constraints are imposed on the joint motions. Using this criterion and the bounded acceleration constraint when impact has been detected, the arm will be slowed and then the end effector will be returned to the point of impact.

\subsection{Minimizing End Effector Distance from Point of Impact}

The types of criterion which can be developed for a manipulator and used with FSP can be divided into two categories: those that are specified in terms of joint space tasks and those that are specified in terms of work space tasks. Minimizing the distance between the end effector and the point of impact falls into the second of these categories. This second category brings up an unfortunate shortcoming of inverse techniques in general and FSP in particular, namely that a closed form solution is not available if the quantities on both sides of Eq. 4 are variable. In order to generate the $m-n+1$ vectors $\bar{g}_{i}, \Delta \bar{x}$ must be known. However, since we want to minimize a quantity which involves a variable size for $\Delta \bar{x}$, we cannot explicitly know $\Delta \bar{x}$ before calculating the $\bar{g}_{i}$ vectors when using this criterion.

In order to avoid the problem mentioned above, $\Delta \bar{x}$ is created by setting its value equal to the distance between the point of impact and the position of the end effector. If any of the components of $\Delta \bar{x}$ are larger than a maximal value, the entire vector is scaled by the largest value so that the largest component is then 0.01 . A least norm criterion is then applied to the joint motions. This method is described more completely below.

\subsection{Bounded Acceleration}

To produce motions which are realistic, each joint of the manipulator must be subject to a finite change in velocity. In other words, for each step of the trajectory, a bounded acceleration constraint must be satisfied. To determine an appropriate formulation for the bounded acceleration constraint, consider a discretization of vector acceleration:

$$
\begin{aligned}
\bar{a} & =\frac{\Delta \bar{v}}{\Delta t} \\
& =\frac{\Delta \bar{q}_{k}-\Delta \bar{q}_{k-1}}{(\Delta t)^{2}}
\end{aligned}
$$

where $k$ signifies the end effector motion which is to be calculated in the current time step and $k-1$ signifies the motion made in the previous time step. If $\Delta t$ is a fixed scalar value, the bounded acceleration for a joint, $i$, may be written as

$$
c_{l}^{i} \leq \Delta \vec{q}_{k}^{i}-\Delta \bar{q}_{k-1}^{i} \leq c_{u}^{i}
$$

where $c_{l}^{i}$ and $c_{u}^{i}$ are constant scalars representing the upper and lower velocity change limits. The imposed constraint for either the upper or lower limit is

$$
C=\Delta \bar{q}_{k}^{i}-\Delta \bar{q}_{k-1}^{i}-c_{u / l}^{i}=0
$$

or,

$$
\frac{\sum_{j=1}^{m-n+1} \bar{g}_{j}^{i} t_{j}}{\Delta \bar{q}_{k-1}^{i}+c_{u / l}^{i}}-1=0
$$

where $c_{u / l}^{i}$ corresponds to the appropriate upper or lower acceleration limit for joint $i$. Expressing the 
constraint in the form $C=\bar{\beta}^{T} \bar{t}-1=0$ gives

$$
\bar{\beta}, \quad \beta_{j}=\frac{\bar{g}_{j}^{i}}{\Delta \bar{q}_{k-1}^{i}+c_{u / l}^{i}}, \quad j=[1, m-n+1]
$$

where $g_{j}^{i}$ is the $i$ th component of vector $\bar{g}_{j}$.

\subsection{Impact Mitigation Algorithm}

The algorithm which is currently being used to slow the manipulator arm and halt the end effector at the point of impact is shown in Fig. 1. The first step after

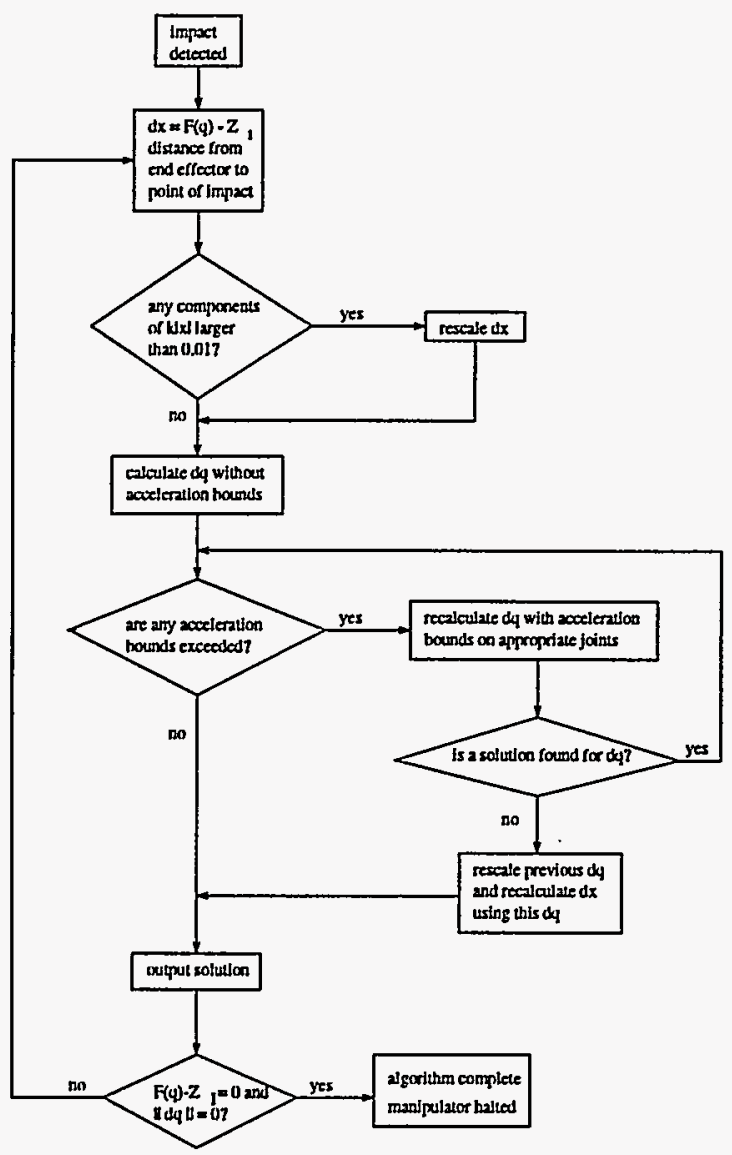

Figure 1: Algorithm for halting robot after impact.

impact is to calculate the distance between the end effector and the point of impact. Immediately after impact, this distance will be almost negligible. However, the joint velocities will almost definitely be too large for the manipulator to be halted in one time step. If any of the components of $\Delta \bar{x}$ are larger than 0.01 (chosen to produce a smooth trajectory), $\Delta \bar{x}$ is rescaled so that the largest component is 0.01 in magnitude. Next the joint motions which would produce this work space motion are calculated with a least norm criterion and no acceleration bound constraint. The components of
$\Delta \bar{q}$ are then checked to see if any of the joint motions would violate the bounded acceleration constraint. If none of the acceleration bounds are violated, the joint motion is passed out to the manipulator to be performed. If some of the joints did violate their acceleration bounds, then $\Delta \bar{q}$ must be recalculated using the bounded acceleration constraint on those joints. This iteration process cannot be avoided since the joint accelerations cannot be known until the joint motions are calculated for the given $\Delta \bar{x}$.

When acceleration bounds are imposed on some of the joints, the possibility exists that the desired $\Delta \bar{x}$ value cannot be produced. In this case, the original value of $\Delta \bar{q}$ is rescaled so that the largest acceleration is inside its bound, and $\Delta \bar{x}$ is recalculated based upon this restricted $\Delta \vec{q}$ vector. If the end effector has not returned to the point of impact, and the joint velocities are not all zero, then iterations through the algorithm shown in the flow chart are made until these conditions are met.

\section{Results of Simulations}

In order to test the impact algorithm, simulations were performed with a simple redundant planar manipulator that has four rotational joints. A planar object has been placed in the workspace of the manipulator. The impact behavior of the manipulator as discussed below was created by specifying a trajectory that passed through the planar object. Images of the starting and initially desired final configurations along with the planar object (seen as a line) are shown in Fig. 2. Values of $\pm 2 \mathrm{deg} / \mathrm{s}^{2}$ were used for the acceleration bounds to exaggerate the post-impact behavior. Joint and end effector motions for a typical impact
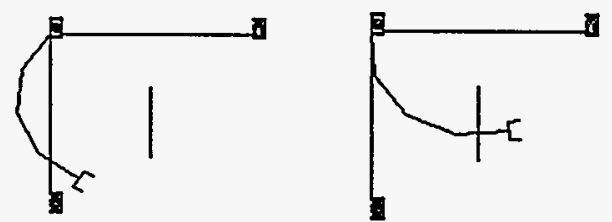

Figure 2: Starting and ending configurations for the simulated manipulator defining the desired trajectory.

trajectory are shown in Figs. 3 and 4 . The motions of both the joints and the end effector show a decaying oscillatory behavior that occurs due to the dual requirement that joint motions and distance between the end effector and the point of impact be minimized. Just after impact, (time step 35) the joints cannot stop or reverse direction quickly, so the end effector moves a 


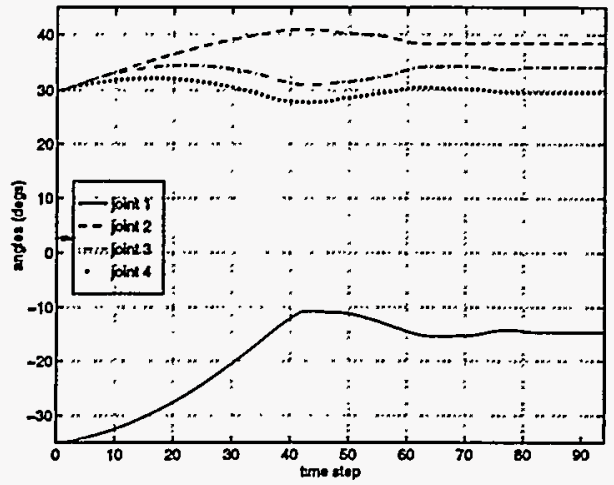

Figure 3: Joint motions for typical impact trajectory.

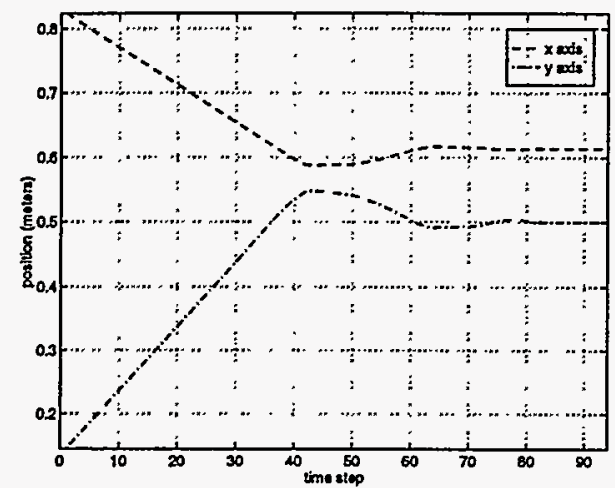

Figure 4: End effector motions for typical impact trajectory.

small distance away from the point of impact, then as the joints are slowed, the end effector begins to move back toward the point of impact. As the manipulator reaches the point of impact, the joints again cannot immediately halt, but their accelerations are smaller, and the overshoot is not as great. The end effector is returned to the point of impact after only a short period of oscillation.

The joint velocities associated with this trajectory are shown in Fig. 5. These plots also show the oscillatory behavior of the trajectory as the arm is slowed and halted. The nonsmooth appearance of these plots is partly due to the discretized nature of the algorithm and partly to the acceleration limits on the joints. If any of the joints reach their limits, the others must compensate and the joint velocities demonstrate a nonsmooth behavior.

Plots of the norm of the error in end effector position and the norm of the joint motion are shown in Figs. 6 and 7. The selected threshold point for an acceptably small value of the distance from the end effector to the point of impact is $1.0 e^{-4}$. The selected

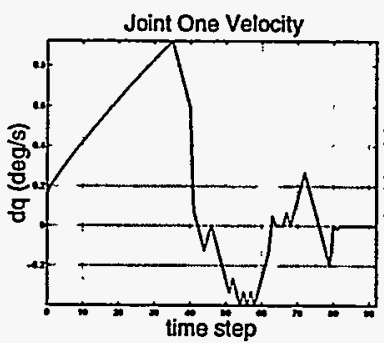

Joint Three Velocity
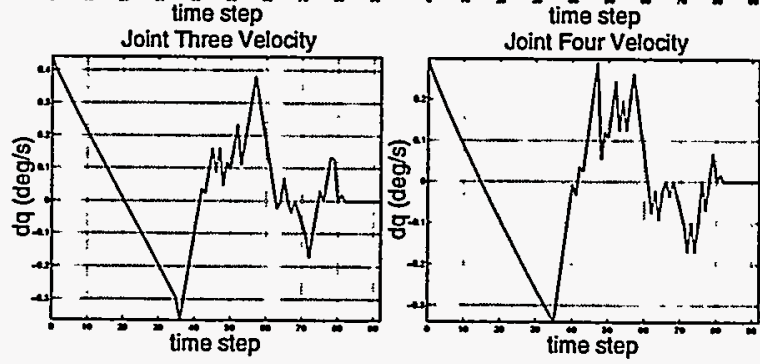

Figure 5: Joint velocities for typical impact trajectory.

value for an acceptably small norm of the joint motions is $1.0 e^{-6}$. These plots also exhibit oscillatory behavior after impact. However, the oscillations are out of phase from each other which illustrates the competition between decreasing distance from the end effector to the point of impact and decreasing norm of the joint angle motions that leads to the demonstrated oscillatory behavior in the previous figures.

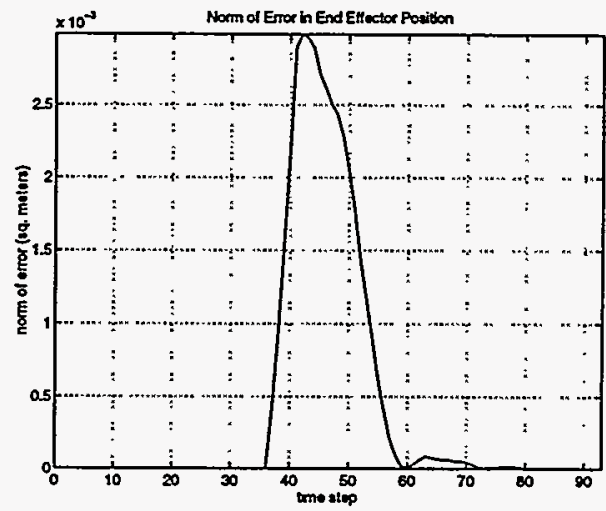

Figure 6: Norm of the error in end effector position.

\section{Conclusion}

An approach to impact mitigation for manipulators with joint acceleration constraints has been presented. Using this constraint with the Full Space $\mathrm{Pa}$ rameterization method has lead to a preliminary algorithm which utilizes the manipulator redundancy to slow down the end effector after an unexpected impact and return it to the point of impact. In the current system, the manipulator follows a planned trajec- 


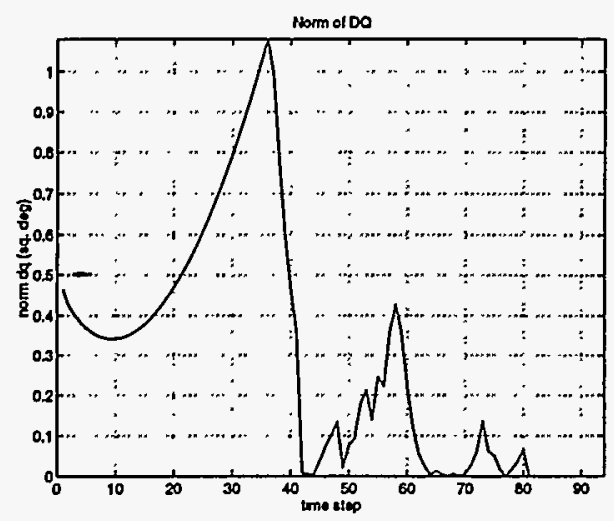

Figure 7: Norm of joint velocities.

tory before impact under a least norm criterion on the joint motions. At the point of impact, the originally planned trajectory is discontinued, and the motion of the end effector is specified with the intention of keeping it as close to the point of impact as possible subject to the bounded acceleration constraint. Without the constraint of bounded acceleration, the end effector would move to the point of impact immediately, exhibiting unrealistically large actuator accelerations which would not be possible with a real robot. The behavior of the redundant manipulator after impact has been illustrated and discussed using a simulation of a simple planar manipulator. The competition between the minimization of end effector displacement and the slowing down of all joints under the acceleration bound constraint is clearly illustrated which leads to an oscillatory motion before the system settles at the point of impact. Future work will include investigation of other optimization criterion to further improve the overall mitigating effect possible with the use of redundancy.

\section{References.}

[1] G. A. Fries, C. J. Hacker and F. G. Pin, "FSP (Full Space Parameterization) version 2.0," Oak Ridge National Laboratory Technical Report No. ORNL/TM-13021, 1995 (available from NTIS, U.S. Depart. of Commerce, 5285 Port Royal Rd., Springfield, VA 22161).

[2] O. Khatib and J. Burdick, "Motion and Force Control of Robot Manipulators," IEEE Conf. on Robotics and Automation, San Francisco, CA, pp. 1381-1386, 1986.

[3] J. Kim, M. W. Gertz, and P. K. Khosla "Exploiting Redundancy to Reduce Impact Force," $J$. of Intel. and Rob. Sys., vol. 9, pp. 273-290, 1994.
[4] K. A. Morgansen and F. G. Pin, "Enhanced Code for the Full Space Parameterization Approach to Solving Underspecified Systems of Algebraic Equations, version 1.0," Oak Ridge National Laboratory Technical Report No. ORNL/TM-12816, 1995, (available from NTIS, U.S. Depart. of Commerce, 5285 Port Royal Rd., Springfield, VA 22161).

[5] D. N. Nenchev, "Redundancy Resolution through Local Optimization: A Review," J. of Rob. Sys., vol. 6 , no. 6, pp. 769-798, 1989.

[6] F. G. Pin et al, "A New Solution Method for the Inverse Kinematics Joint Velocity Calculations of Redundant Manipulators," IEEE Conf. on Robotics and Automation, San Diego, CA, pp. 96-102, 1994.

[7] V. Potkonjak, "Application of Redundant Robots to Constrained-Motion Tasks," Robotica, vol. 10, pp. 397-407, 1992.

[8] B. Siciliano, "Kinematic Control of Redundant Robot Manipulators: A Tutorial," J. of Intel. and Rob. Sys., vol. 3, pp. 201-212, 1990.

[9] K. Youcef-Toumi and D. A. Gutz, "Impact and Force Control," IEEE Conf. on Robotics and Automation, Scottsdale, AZ, pp. 410-416, 1989.

[10] M. Vukobratovic and V. Potkonjac, "Constrained Gripper Motion in Assembly Manipulation," J. of Rob. Sys., vol. 3, no. 3, pp. 335-347, 1986.

[11] I. D. Walker, "Impact Configurations and Measures for Kinematically Redundant and Multiple Armed Robot Systems," IEEE Trans. on Robotics and Automation, Vol. 10, No. 5, pp. 670683, Oct. 1994.

[12] Y. Wang and M. T. Mason, "Modeling Impact Dynamics for Robotic Operations," IEEE Conf. on Robotics and Automation, Raleigh, NC, pp. 678-685, 1987.

[13] Y. Zheng and H. Hemami, "Impact Effects of Biped Contact with the Environment", IEEE Trans. Systems, Man and Cyber., vol. SMC-14, No. 3, pp. 437-443, May/June 1984.

[14] Y. Zheng and H. Hemami, "Mathematical Modeling of a Robot Collision with its Environment," J. of Rob. Sys., vol. 2, No. 3, pp. 289-307, 1985. 